REPERTORIO BIBLIOGRÁFICO SOBRE LA ACCIÓN DE AMPARO EN VENEZUELA

JUAN MANUEL GOIG MARTÍNEZ 



\section{REPERTORIO BIBLIOGRÁFICO SOBRE LA ACCIÓN DE AMPARO EN VENEZUELA}

POR

JUAN MANUEL GOIG MARTÍNEZ

Actas de la Comisión redactora del Proyecto de Constitución, Congreso de la República, tomo I, Caracas, 1959-60.

Actas del Primer Congreso Mexicano y Segundas Jornadas Latinoamericanas de Derecho Procesal, México, 1960.

Agudo Freytes, Esteban: "Estado actual del Amparo en Venezuela», Libro Homenaje a Rafael Caldera, UCV, tomo II, Caracas. 1979.

- "Estudios sobre la Constitución», Libro Homenaje a Rafael Caldera, UCV, tomo II, Caracas, 1979.

- "La garantía de Amparo", Recopilación del Ministerio Público, tomo l, Caracas, 1978.

- "Algunos casos de Amparo y Habeas Corpus", Anuario del Colegio de Abogados del Estado Lara, Barquisimeto, 1969.

AlCALÁ ZAMORA, Niceto: «Enseñanzas y sugerencias de algunos tratadistas latinoamericanos sobre la acción", Estudios de Derecho Procesal. Libro Homenaje a Hugo Alsina, Buenos Aires, 1946.

Álvarez Martinez, Víctor: «Recurso de Amparo y Habeas Corpus en Venezuela", Academia de Ciencias Políticas y Sociales, número 2, Caracas, 1982.

Andueza AcuÑA, José G.: La jurisdicción constitucional en el derecho venezolano, Caracas, 1974.

Antequera Padilla, Ricardo: El derecho a la protección de la Salud, Instituto de Estudios Jurídicos del Estado Lara, Barquisimeto, 1987. 
Anuario del Colegio de Abogados del Estado Lara, Barquisimeto, 1969.

Ávila Ferrer, Marcelino: "La Constitución de Venezuela y los Derechos Humanos", Ministerio Público, número X, enero-abril, 1983.

Ayala Corao, Carlos M.: La Ley Orgánica de Amparo sobre Derechos y Garantías constitucionales, Caracas, 1988.

Araujo Juárez, José: Sinopsis doctrinal sobre los principios constitucionales de Amparo, Instituto de Estudios Jurídicos del Estado Lara, Barquisimeto, 1987.

Barbi Agrícola: El Mandato de Seguridad, Bello Horizonte, Brasil, 1961.

Bertolino, Pedro: La cosa Juzgada en el Amparo, Editorial Perrot, Buenos Aires, 1968.

Bidart Campos: Régimen Legal y Jurisprudencial del Amparo, Buenos Aires, 1968.

Borea OdIA, Alberto: La defensa constitucional: El Amparo, Lima, 1977.

Borjas Arminio: Comentarios al Código de Procedimiento Civil, tomo III, Caracas, 1987.

Bravo Bravo, Héctor: El Amparo frente al fraude procesal, Instituto de Estudios Jurídicos del Estado Lara, Barquisimeto, 1987.

Brewer Carias, Allan R.: Constitución de 1961, Edición Jurídica Venezolana, Caracas, 1984.

- Estado de Derecho y Control Judicial, Instituto de Administraciones Públicas, Madrid, 1987.

- Garantías constitucionales de los derechos del hombre, Caracas, 1976.

- La Ley de Amparo, Editorial Jurídica Venezolana, Caracas, 1988.

- Ley Orgánica de Amparo sobre Derechos y Garantías Constitucionales, Editorial Jurídica Venezolana, Caracas, 1988.

- "Amparo sobre Amparo", Revista de Derecho Público, número 34, abril-junio, Caracas, 1988.

- Instituciones Políticas y Constitucionales, Caracas, 1978.

- Las Constituciones de Venezuela, Centro de Estudios Constitucionales, Madrid, 1985. 
- El control de constitucionalidad de los actos estatales, Caracas, 1977.

BrICE, Ángel F.: «El juicio de Amparo y el Anteproyecto de Constitución de 1946", Boletin de la Academia de Ciencias Políticas y Sociales, tomo XI, Caracas, 1970.

- "Las libertades individuales y las formas de protegerlas", Revista del Colegio de Abogados del Estado Zulia, 1939.

Briceño, Gustavo: Comentarios a la Ley de Amparo, Editorial Kinesis, Ca. racas, 1991.

BriceÑo, León Humberto: La acción de inconstitucionalidad en Venezuela, Caracas, 1989.

BurgoA, Ignacio: El juicio de Amparo, Editorial Porrúa, México, 1975.

CARDozo, Manuel. "El recurso de Amparo: Nuevo enfoque jurisprudencial», El Universal, 3 de diciembre de 1982.

Castro, Juventino V.: Lecciones de garantías y amparo, México, 1978.

Congreso de la República: La Constitución de 1961 y evolución constitucional en Venezuela, Caracas, 1971.

Cordero Giusti, Jesús: El amparo y la justicia Laboral, Instituto de Estudios Jurídicos del Estado Lara, Barquisimeto, 1987.

Corporación Publicitaria Nacional: La Constitución Federal de Venezuela de 1811, Caracas, 1961.

Chissione, Tulio: «La garantía de amparo judicial», Diario Judicial, Caracas, 1972.

Dana Montaño, S.: "La reglamentación del amparo de los derechos y garantías", Revista La Ley, Buenos Aires, 1966.

De Miguel, José Antonio: Amparo y habeas corpus en la Constitución de 1961, Caracas, 1965.

De SolA, René: «Plena vigencia del recurso de Amparo», Revista del Consejo de Judicatura, año VIII, número 31, octubre-diciembre de 1983, Caracas.

- El recurso de amparo en Venezuela, Instituto de Estudios Jurídicos del Estado Lara, Barquisimeto, 1987. 
- Consideraciones sobre el recurso de amparo, Instituto de Estudios Jurídicos del Estado Lara, Barquisimeto, 1987.

Diario de Tribunales del Estado Lara: "El amparo constitucional en Venezuela", Recopilación de Sentencias, tomo II, Barquisimeto, 1987.

Duque Corredor, Ramón: "El proceso de acción de amparo constitucional», Academia de Ciencias Políticas y Sociales, número 2, Caracas, 1989.

Duque Sánchez, José Ramón: Procedimientos especiales contenciosos, Caracas, 1981.

DURÁn LEón, Gloria: El recurso de amparo en el ámbito laboral, Instituto de Estudios Jurídicos del Estado Lara, Barquisimeto, 1987.

Escala Zerpa, Reinaldo: Libertad de leer, Editorial La torre, Caracas, 1968.

- Recurso de amparo contra arbitrariedad de funcionarios públicos, Editorial La torre, Caracas, 1968.

- "Amparo. Tribunales y Constitución", Ministerio Público, 2. etapa, número IX, septiembre-diciembre de 1982, Caracas.

Escarrá Malave, Hernán: "El amparo en Venezuela», Ponencias Primer Seminario Latinoamericano de Jurisdicción constitucional, Chile, 1987.

- El amparo constitucional y el procedimiento de iniciativa popular, Instituto de Estudios Jurídicos del Estado Lara, Barquisimeto, 1987.

- Razón Histórica y Razón política, Caracas, 1977.

Escobar Salom, Ramón: El amparo en Venezuela, Caracas, 1971.

Esouivel Obregón, Toribio: Influencias estadounidenses y españolas en México, México, 1952.

Fiscalía General de la República: Informe al Congreso, años 1966 y 1969, Caracas.

FIX ZAMUDIO, Héctor: La protección procesal de los derechos humanos ante las jurisdicciones nacionales, Universidad Autónoma de México, $\mathrm{Ci}$ vitas, España, 1982.

- "Diversos significados del amparo en el derecho latinoamericano", Boletín informativo de Derecho Comparado, México, 1965.

- "El amparo en México y Venezuela", UCV, Libro Homenaje a la memoria de Lorenzo Herrera Mendoza, Caracas, 1972. 
- Mandato de Seguridad y Juicio de amparo, Instituto de Derecho Comparado, México, 1970.

- "Algunos problemas que plantea el amparo contra leyes", Boletín informativo de Derecho Comparado, número 37, México, 1960.

- "Reflexiones sobre la naturaleza procesal del amparo", Revista de la Facultad de Derecho de México, número 56, México, 1964.

- «El juicio de amparo en Latinoamérica», Memoria del Colegio Nacional, México, 1978.

Fortul GIL: Historia Constitucional en Venezuela, tomo I, Caracas, 5. edición.

GARAY, Juan: Legislación Laboral práctica, Editorial Garay, Caracas, 1991.

Grant, J. A. C.: El control jurisdiccional de la constitucionalidad, México, 1963.

Grisanti Aveledo, Hernando: El amparo de la libertad personal, Instituto de Estudios Jurídicos del Estado Lara, Barquisimeto, 1987.

Gros EsPIEL, H.: Estudios sobre derechos humanos, tomo ll, coedición con el Instituto Interamericano de Derechos Humanos, Madrid, 1988.

Guzmán Fernández: Código práctico de Procedimiento Civil, Caracas, 1987.

LANz DuRET: Derecho constitucional venezolano, México, 1931.

LA Roche, Humberto: El control jurisdiccional de la constitucionalidad en Venezuela y EE.UU., Maracaibo, 1972.

Leyes y Decretos Reglamentarios de Venezuela, tomo VI, Caracas, 1943.

Linares Benzo, Gustavo: El proceso de Amparo en Venezuela, Caracas, 1987.

Mariñas Otero, Luis: Las Constituciones en Venezuela, Editorial Cultura Hispánica, Madrid, 1965.

MARTínez AM., Guaicaipuro: Acción de amparo y legitimación pasiva, Doctrina de la Procuraduria General de la República, Caracas, 1972.

MendozA, J. Rafael: El juez civil en el recurso de amparo, Instituto de Estudios Jurídicos del Estado Lara, Barquisimeto, 1987.

- Casos de jurisprudencia o de nuevas interpretaciones, Barquisimeto, 1973. 
Molina Galicia, René: El Amparo a Redondela, Caracas, 1984.

Morales Bello, David: El amparo constitucional, Instituto de Estudios Jurídicos del Estado Lara, Barquisimeto, 1987.

- Acción Democrática y la Ley Orgánica de la Corte Suprema, Caracas, 1976.

Morris Sierralta: De los recursos de Amparo y Habeas Corpus en el Derecho Constitucional Venezolano, Caracas, 1971.

NiKKen, P.: La protección internacional de los derechos humanos. Su desarrollo progresivo, Civitas, coedición con el Instituto Interamericano de Derechos Humanos, Madrid, 1987.

Noriega Catú, Alfonso: La consolidación del juicio de amparo, México, 1980.

Olaso, Luis María: El derecho de amparo, Instituto de Estudios Jurídicos del Estado Lara, Barquisimeto, 1987.

Oropeza, Ambrosio: La nueva Constitución de 1961, Caracas, 1971.

- Evolución constitucional de nuestra República y otros textos, Caracas, 1985.

MARín Gómez, Otto: La protección procesal de las garantías constitucionales en Venezuela: Amparo y Habeas Corpus, Colección Ciencias Jurídicas y Políticas, tomo XI, Caracas, 1989.

Palacios, J. Ramón: Instituciones de Amparo, Puebla, 1963.

Plaz Brual, René: Los derechos Humanos y el Amparo constitucional, Estudios Jurídicos del Estado Lara-Colegio de Abogados del Estado Lara, Barquisimeto, 1988.

- Reflexiones sobre el amparo constitucional y los derechos del hombre, Academia de Ciencias Políticas y Sociales, Caracas, 1989.

Picón Rivas, Ulises: Índice constitucional de Venezuela, Editorial Élite, Caracas, 1944,

Polanco Alcántara, Tomás: Recurso de amparo en la legislación venezolana, Academia de Ciencias Políticas y Sociales, Caracas, 1989.

Quintero, Ramón: «El recurso de amparo", Revista de la Facultad de Derecho de la Universidad Andrés Bello, número 9, Caracas, 1969-70. 
RABASA, Emilio: El juicio constitucional, México, 1955.

Riera Encinoza, Argenis: Sumario sobre la Ley Orgánica de Amparo, Caracas, 1988.

- Jurisprudencia sobre amparo, Caracas, 1988.

Rios Espinoza, Alejandro: Amparo y Casación, México, 1960.

Romero Mucl, Humberto: El amparo constitucional por omisión o retardo, Biblioteca de la Academia de Ciencias Políticas y Sociales, Caracas, 1989.

Rondón DE SANSó, Hildegard: Amparo Constitucional, Caracas, 1988.

- "Amparo Constitucional", Revista de Derecho Público, número 21, Caracas, 1985.

- Análisis crítico de la Ley Orgánica de Amparo, Academia de Ciencias Políticas y Sociales, Caracas, 1989.

- "Consideraciones generales sobre el recurso de amparo", Revista de Derecho Público, número 21, Caracas, 1985.

Rosell Seuheun, J. L.: La función política del órgano judicial, Instituto de Estudios Jurídicos del Estado Lara, Barquisimeto, 1987.

SACHICA, Luis Carlos: El control de constitucionalidad, Bogotá, 1980.

SANTANA Música, Miguel: ¿Está o no vigente el amparo constitucional?, Instituto Jurídico del Estado Lara, Barquisimeto, 1987.

- ¿Es necesaria una Ley de amparo?, Instituto de Estudios Jurídicos del Estado Lara, Barquisimeto, 1987.

- El Amparo y la Corte Suprema, Instituto de Estudios Jurídicos del Estado Lara, Barquisimeto, 1987.

- La casación venezolana dictamina la plena vigencia del amparo, Caracas, 1987.

Sarmiento Núñez, J. Gabriel: Temas Jurídicos, Caracas, 1972.

Solana, Fabio: "Amparo contra la Universidad de Carabobo", Diario de Tribunales, número 5039, año XV, 10 de mayo de 1991.

Suárez Santiago, Gerardo: Diccionario de la Constitución, Caracas, 1978. 
TAMAYO, Luis Alejandro: Recurso de amparo y recurso de casación, Instituto de Estudios Jurídicos del Estado Lara, Barquisimeto, 1987.

Tovar Tamayo, Orlando: "El marco jurídico del amparo en el derecho constitucional comparado", Revista de la Facultad de Ciencias Jurídicas y Sociales, número 70, Caracas, 1988.

- "La jurisdicción constitucional", Academia de Ciencias Políticas y Sociales, número 10, Caracas, 1981.

VILORA HERnÁNDEZ, Gilmer: Los derechos humanos en Venezuela, Estado de Valera, 1979.

Wolf, Ernesto: Tratado de Derecho constitucional venezolano, tomo II, Caracas, 1945.

Zavala, Juan O.: Las Constituciones vigentes en América, tomo I, volumen 1, Caracas, 1961.

\section{Recopilaciones jurisprudenciales}

Bustamante Miranda, Maruja: 15 años de jurisprudencia de la Corte Suprema de Justicia, Universidad Central, Caracas, 1978.

Jurisprudencia de la Corte Suprema de Justicia, Publicaciones del Ministerio de Justicia.

Boletín de Jurisprudencia de la Corte Suprema de Justicia.

Repertorio cronológico de Legislación y Jurisprudencia, Editorial Leyes de Venezuela, Caracas. Director: José Marín Gutiérrez.

Jurisprudencia analítica de Venezuela. Director: Luis T. Laya. Organización para las investigaciones del Derecho, Caracas.

Jurisprudencia venezolana. Director: Arquímedes E. González, Caracas.

TAPIA, Óscar Pierre: Jurisprudencia de la Corte Suprema de Justicia.

- Jurisprudencia de la Corte Primera de lo Contencioso-Administrativo.

- Jurisprudencia de los Tribunales de última instancia.

- Jurisprudencia de los Tribunales superiores de lo Contencioso-tributario.

- Jurisprudencia de Tránsito, de lo Contencioso-administrativo y de Menores.

- Jurisprudencia de Tránsito, de lo Contencioso-tributario y de Menores. 